\title{
Spreading of Wave Packets in the Anderson Model on the Bethe Lattice
}

\author{
Abel Klein ${ }^{\star}$ \\ Department of Mathematics, University of California, Irvine, CA 92717-3875, USA. \\ E-mail: aklein@math.uci.edu
}

Received: 5 April 1995

\begin{abstract}
The spreading of wave packets evolving under the Anderson Hamiltonian on the Bethe Lattice is studied for small disorder. The mean square distance travelled by a particle in a time $t$ is shown to grow as $t^{2}$ for large $t$.
\end{abstract}

\section{Introduction}

The Anderson model [6] gives a description of the motion of a quantum-mechanical electron in a crystal with impurities. It is given by the random Schrödinger operator

$$
H_{\lambda}=\frac{1}{2} \Delta+\lambda V \text { on } l^{2}(\mathbb{L}) ;
$$

where $\mathbb{L}$ is either $\mathbb{Z}^{d}$ or the Bethe lattice $\mathbb{B}$ (same as Cayley tree-an infinite connected graph with no closed loops and a fixed number $K+1$ of nearest neighbors at each vertex $(K \geqq 2$, so $\mathbb{B}$ is not the line $\mathbb{R})$; the distance between two sites $x$ and $y$ in $\mathbb{B}$ will be denoted by $d(x, y)$ and is equal to the length of the shortest path connecting $x$ and $y$ ). The (centered) Laplacian $\Delta$ is defined by

$$
(\Delta u)(x)=\sum_{y} u(y),
$$

where the sum runs over all nearest neighbors of $x$ in $\mathbb{L}$, and $V$ is a random potential, with $V(x), x \in \mathbb{L}$, being independent, identically distributed random variables with common probability distribution $\mu$. The real parameter $\lambda$ is called the disorder.

It follows from ergodicity that the spectrum of the Hamiltonian $H_{\lambda}$ is given by

$$
\sigma\left(H_{\lambda}\right)=\sigma\left(\frac{1}{2} \Delta\right)+\lambda \operatorname{supp} \mu
$$

with probability one $[33,9,3]$, where $\sigma\left(\frac{1}{2} \Delta\right)$ equals $[-d, d]$ if $\mathbb{L}=\mathbb{Z}^{d}$ and $[-\sqrt{K}$, $\sqrt{K}]$ if $\mathbb{L}=\mathbb{B}$. The decomposition of $\sigma\left(H_{\lambda}\right)$ into pure point spectrum, absolutely

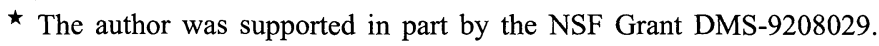


continuous spectrum and singular continuous spectrum is also independent of the choice of $V$ with probability one $[26,9,3]$. In the physics literature $([6,32,37$, $1,2,29]$ and others) one finds the following picture: In one and two dimensions, as long as the potential is random (i.e., $\lambda \neq 0$ ), the model shows exponential localization (i.e., pure point spectrum with exponentially decaying eigenfunctions). In three and more dimensions both localized and extended states (i.e., absolutely continuous spectrum) are expected for small disorder, with the energies of extended and localized states being separated by the mobility edge; the model exhibits the Anderson metal-insulator transition. In the Bethe lattice the picture is similar to the multidimensional case $([1,2,27,31,30]$ and others), with perhaps a mobility interval instead of a mobility edge (see the discussion in [22]).

An alternative way to distinguish between localized and extended states is by looking at their time evolution. Let us consider a particle which is localized at the origin 0 of $\mathbb{L}$ (we fix an origin 0 for the Bethe lattice) at time $t=0$, at time $t$ it will be described by the wavefunction $\psi_{t}^{(\lambda)}=\mathrm{e}^{i t H_{\lambda}} \delta_{0}$, with $\delta_{0}$ being the delta function at $x=0$. A measure of the spread of the wave packet at time $t$ is given by its mean square displacement

$$
r_{\lambda}^{2}(t)=\sum_{x \in \mathbb{L}}|x|^{2}\left|\psi_{t}^{(\lambda)}(x)\right|^{2}=\sum_{x \in \mathbb{L}}|x|^{2}\left|\mathrm{e}^{i t H_{\lambda}}(0, x)\right|^{2},
$$

where $|x|=d(0, x)$ (it is not a norm in $\mathbb{B}$ ) and $\mathrm{e}^{i t H_{\lambda}}(0, x)=\left\langle\delta_{0}, \mathrm{e}^{i t H_{\lambda}} \delta_{x}\right\rangle$. We always have ([35], we give the argument in Lemma A.1)

$$
r_{\lambda}^{2}(t) \leqq C t^{2} \text { with } C=\left\|\frac{1}{2} \Delta\right\|^{2}= \begin{cases}d^{2} & \text { if } \mathbb{L}=\mathbb{Z}^{d} \\ K & \text { if } \mathbb{L}=\mathbb{B}\end{cases}
$$

In the case of free motion $(\lambda=0)$ one has $r_{\lambda}^{2}(t) \approx$ const $t^{2}$. Anderson [6] argued that for large disorder, there are only localized states so the particle localizes near the origin, i.e., $\sup _{t} r_{\lambda}^{2}(t)<\infty$ with probability one (see the discussion in [14]). In three or more dimensions, the particle should diffuse with probability one for small disorder, and one should have $[14,28] \mathbb{E}\left(r_{\lambda}^{2}(t)\right) \approx$ const $t$, where $\mathbb{E}$ denotes expectation with respect to the probability distribution of the random potential. On the Bethe lattice, however, we should expect $\mathbb{E}\left(r_{\lambda}^{2}(t)\right) \approx$ const $t^{2}$ at small disorder, since on the Bethe lattice both free motion and what should be diffusive motion (e.g., the simple random walk) have mean square displacements growing as $t^{2}$.

There are now many proofs of exponential localization for the Anderson Hamiltonian: for any disorder in one dimension (e.g., [15,26, 8, 11] and others), and for large disorder or low energy in the multidimensional case (e.g., $[14,13,10,36,28,11,5,4,19,16,12]$ and others) and on the Bethe lattice $[5,4,16]$. When the single site potential distribution $\mu$ has a bounded density and compact support, it is also proven that $\mathbb{E}\left(\sup _{t} r_{\lambda}^{2}(t)\right)<\infty$ for large disorder, both in the multidimensional case [28,4] and on the Bethe lattice [4].

There are presently no rigorous results about extended states in $\mathbb{Z}^{d}$. In previous work $[20,21,23]$ we proved the existence of extended states on the Bethe lattice for small disorder. In this article we show that

$$
\limsup _{t \rightarrow \infty} \mathbb{E}\left(\frac{r_{\lambda}^{2}(t)}{t^{2}}\right)>0
$$


on the Bethe lattice, for sufficiently small disorder. Combining this result with the uniform deterministic bound given by (1.5), we conclude (see Lemma D.1) that

$$
\mathbb{P}\left(\limsup _{t \rightarrow \infty} \frac{r_{\lambda}^{2}(t)}{t^{2}}>0\right)>0
$$

for the Anderson model on the Bethe lattice with small disorder. It follows from ergodicity that

where

$$
\mathbb{P}\left(\limsup _{t \rightarrow \infty} \frac{r_{x, \lambda}^{2}(t)}{t^{2}}>0 \text { for some } x \in \mathbb{B}\right)=1,
$$

$$
r_{x, \lambda}^{2}(t)=\sum_{y \in \mathbb{B}} d(x, y)^{2}\left|\left(\mathrm{e}^{i t H_{\lambda}} \delta_{x}\right)(y)\right|^{2}
$$

We will now describe our results in more detail. Our assumption on $\mu$ is that its characteristic function $h(t)=\int e^{-i t v} d \mu(v)$ is differentiable on $(0, \infty)$, with $h^{\prime}(t)$ absolutely continuous, $h^{\prime}(t)$ and $h^{\prime \prime}(t)$ bounded on $(0, \infty)$. These conditions are satisfied by any probability distribution $\mu$ with a finite second moment (e.g., uniform, Gaussian or Bernoulli distributions) and by the Cauchy distribution. Our main result is:

Theorem 1.1. Let $\mathbb{L}=\mathbb{B}$, then for sufficiently small $\lambda$ we have

$$
\liminf _{t \rightarrow \infty} \frac{1}{t^{3}} \int_{0}^{t} \mathbb{E}\left(r_{\lambda}^{2}(s)\right) d s>0 .
$$

In particular, we have (1.6), (1.7) and (1.8).

Notice that (1.6) follows immediately from (1.10). From (1.5) we know the uniform deterministic bound:

Thus we also get

$$
\frac{1}{t^{3}} \int_{0}^{t} r_{\lambda}^{2}(s) d s \leqq \frac{K}{3} \quad \text { for any } t
$$

$$
\mathbb{P}\left(\limsup _{t \rightarrow \infty} \frac{1}{t^{3}} \int_{0}^{t} r_{\lambda}^{2}(s) d s>0\right)>0
$$

from (1.10) by Lemma D.1. Ergodicity again gives

$$
\mathbb{P}\left(\limsup _{t \rightarrow \infty} \frac{1}{t^{3}} \int_{0}^{t} r_{x, \lambda}^{2}(s) d s>0 \text { for some } x \in \mathbb{B}\right)=1 .
$$

This paper is organized as follows: In the next section we reformulate the problem in terms of Green's functions; Theorem 1.1 is shown to follow from Theorem 2.2. A review of the mathematical setting we use is given in Sect. 3. Theorem 2.2 is proven in Sect. 4. The appendices contain some results needed in our proofs.

\section{Green's Functions}

We will now reformulate Theorem 1.1 in terms of the Green's function $G_{\lambda}(x, y ; z)=$ $\left\langle\delta_{x},\left(H_{\lambda}-z\right)^{-1} \delta_{y}\right\rangle$. We start with the well known relation (see Lemma A.2)

$$
\int_{0}^{\infty} \mathrm{e}^{-\eta t} \mathbb{E}\left(r_{\lambda}^{2}(t)\right) d t=\frac{1}{2 \pi} \int_{-\infty}^{\infty}\left\{\sum_{x \in \mathbb{L}}|x|^{2} \mathbb{E}\left(\left|G_{\lambda}\left(0, x ; E+i \frac{\eta}{2}\right)\right|^{2}\right)\right\} d E,
$$


valid for any $\eta>0$. Similarly to (A.8), (A.9) and (A.10), we always have

$$
\begin{gathered}
\int_{-\infty}^{\infty} \mathbb{E}\left(\left|G_{\lambda}\left(0, x ; E+i \frac{\eta}{2}\right)\right|^{2}\right) d E \leqq \frac{2 \pi}{\eta} \\
\int_{-\infty}^{\infty}\left\{\sum_{x \in \mathbb{L}}|x|^{2} \mathbb{E}\left(\left|G_{\lambda}\left(0, x ; E+i \frac{\eta}{2}\right)\right|^{2}\right)\right\} d E \leqq \frac{4 \pi}{\eta^{3}}\left\|\frac{1}{2} \Delta\right\|^{2},
\end{gathered}
$$

and

$$
\sup _{E \in \mathbb{R}} \sum_{x \in \mathbb{L}}|x|^{2} \mathbb{E}\left(\left|G_{\lambda}\left(0, x ; E+i \frac{\eta}{2}\right)\right|^{2}\right) \leqq \frac{4}{\eta^{4}}\left\|\frac{1}{2} \Delta\right\|^{2} .
$$

By a Tauberian Theorem (e.g., [34, Theorem 10.3]; notice the proof is also valid for lim inf), Theorem 1.1 follows from (2.1), (2.3) and

Theorem 2.1. Let $\mathbb{L}=\mathbb{B}$, then for sufficiently small $\lambda$ we have

$$
\liminf _{\eta \downarrow 0} \eta^{3} \int_{-\infty}^{\infty}\left\{\sum_{x \in \mathbb{B}}|x|^{2} \mathbb{E}\left(\left|G_{\lambda}(0, x ; E+i \eta)\right|^{2}\right)\right\} d E>0 .
$$

By Fatou's lemma, Theorem 2.1 follows immediately from the next theorem.

Theorem 2.2. Let $\mathbb{L}=\mathbb{B}$, then there exists $\lambda_{0}>0$, such that for any $\lambda$ with $|\lambda|<\lambda_{0}$ we can find energies $E_{\lambda}^{ \pm} \in(-\sqrt{K}, \sqrt{K})$, with $\lim _{\lambda \rightarrow 0} E_{\lambda}^{ \pm}= \pm \sqrt{K}$, such that $H_{\lambda}$ has purely absolutely continuous spectrum in the interval $I_{\lambda}=\left(E_{\lambda}^{-}, E_{\lambda}^{+}\right)$, and

$$
\liminf _{\eta \downarrow 0} \eta^{3} \sum_{x \in \mathbb{B}}|x|^{2} \mathbb{E}\left(\left|G_{\lambda}(0, x ; E+i \eta)\right|^{2}\right)>0 \text { for all } E \in I_{\lambda} \text {. }
$$

Purely absolutely continuous spectrum was proved in [20,21], in the remainder of this article we will prove (2.6).

Another quantity that has been used to distinguish between localized and extended states is

$$
D_{\lambda}(E)=\lim _{\eta \downarrow 0} \eta^{2} \sum_{x \in \mathbb{L}}|x|^{2} \mathbb{E}\left(\left|G_{\lambda}(0, x ; E+i \eta)\right|^{2}\right) .
$$

$D_{\lambda}(E)$ is related to the D.C. conductivity by a Kubo-like formula [29, 14]. The belief is that $D_{\lambda}(E)=0$ in the localization region and $D_{\lambda}(E)>0$ in the region of extended states. The usual proofs of localization (e.g., $[14,13,11,5,4]$ ) all show $D_{\lambda}(E) \equiv 0$ in the energy intervals where they give localization. For the Bethe lattice (2.6) gives

Corollary 2.3. Let $\mathbb{L}=\mathbb{B}$, and let $\lambda_{0}, I_{\lambda}$ be as in Theorem 2.2. Then $D_{\lambda}(E) \equiv \infty$ in $I_{\lambda}$.

\section{The Mathematical Setting}

We fix an arbitrary site in $\mathbb{B}$ which we will call the origin and denote by 0 . Given two nearest neighbors sites $x, y \in \mathbb{B}$, we will denote by $\mathbb{B}^{(x \mid y)}$ the lattice obtained by removing from $\mathbb{B}$ the branch emanating from $x$ that passes through $y$; if we do 
not specify which branch was removed we will simply write $\mathbb{B}^{(x)}$. Each vertex in $\mathbb{B}^{(x)}$ has degree $K+1$, with the single exception of $x$ which has degree $K$.

Let $G_{\lambda}^{(0)}(x, y ; z)$ denote the Green's function of the operator $H_{\lambda}$ restricted to $\mathbb{B}^{(0)}$ with Dirichlet boundary conditions; we will write $G_{\lambda}(z)$ and $G_{\lambda}^{(0)}(z)$ for $G_{\lambda}(0,0 ; z)$ and $G_{\lambda}^{(0)}(0,0 ; z)$, respectively. For any $\lambda \in \mathbb{R}, z=E+i \eta$ with $E \in \mathbb{R}$ and $\eta>0$, we define

$$
\begin{aligned}
\xi_{\lambda, z}\left(\varphi_{+}^{2}, \varphi_{-}^{2}\right) & =\mathbb{E}\left(\exp \left\{\frac{i}{4}\left(G_{\lambda}^{(0)}(z) \varphi_{+}^{2}-\overline{G_{\lambda}^{(0)}(z)} \varphi_{-}^{2}\right)\right\}\right) \\
& =\mathbb{E}\left(\exp \left\{\frac{1}{4}\left[i \mathscr{R}_{\lambda}^{(0)}(z)\left(\varphi_{+}^{2}-\varphi_{-}^{2}\right)-\mathscr{I}_{\lambda}^{(0)}(z)\left(\varphi_{+}^{2}+\varphi_{-}^{2}\right)\right]\right\}\right),
\end{aligned}
$$

where $\mathscr{R}_{\lambda}^{(0)}(z)+i \mathscr{I}_{\lambda}^{(0)}(z)$ is the decomposition of $G_{\lambda}^{(0)}(z)$ into its real and imaginary parts; here $\varphi_{ \pm} \in \mathbb{R}^{2}$ with $\varphi_{ \pm}^{2}=\varphi_{ \pm} \cdot \varphi_{ \pm}$. Notice $\mathscr{I}_{\lambda}^{(0)}(z)>0$ since $\eta>0$.

If $\lambda=0$ we can calculate $G_{0}^{(0)}(z)$ obtaining [3]

$$
\xi_{0, z}\left(\varphi_{+}^{2}, \varphi_{-}^{2}\right)=\mathrm{e}^{\frac{i}{2 K}\left\{\left(-z+\sqrt{z^{2}-K}\right) \varphi_{+}^{2}-\overline{\left(-z+\sqrt{z^{2}-K}\right)} \varphi_{-}^{2}\right\}},
$$

where we always make the choice $\operatorname{Im} \sqrt{ }>0$. If $|E|<\sqrt{K}$, we have the pointwise limit

$$
\xi_{0, E}\left(\varphi_{+}^{2}, \varphi_{-}^{2}\right) \equiv \lim _{\eta \downarrow 0} \xi_{0, z}\left(\varphi_{+}^{2}, \varphi_{-}^{2}\right)=\mathrm{e}^{\frac{1}{2 K}\left\{-i E\left(\varphi_{+}^{2}-\varphi_{-}^{2}\right)-\sqrt{K-E^{2}}\left(\varphi_{+}^{2}+\varphi_{-}^{2}\right)\right\}}
$$
of

We now introduce the Banach spaces $\mathscr{K}_{p}, 1 \leqq p \leqq \infty$, given by the completion

$$
\left\{g:[0, \infty) \times[0, \infty) \rightarrow \mathbb{C} \text { of class } C^{2} ;\|g\|_{\mathscr{K}} \equiv\|\|\left\|_{2}+\right\|\|\|_{p}<\infty\right\},
$$

where

$$
\begin{aligned}
& \|\| g\left\|_{p}^{2}=\right\| g\left(\varphi_{+}^{2}, \varphi_{-}^{2}\right)\left\|_{L^{p}\left(\mathbb{R}^{2} \times \mathbb{R}^{2}, d^{2} \varphi_{+} d^{2} \varphi_{-}\right)}^{2}+\right\| 2 \partial_{+} g\left(\varphi_{+}^{2}, \varphi_{-}^{2}\right) \|_{L^{p}\left(\mathbb{R}^{2} \times \mathbb{R}^{2}, d^{2} \varphi_{+} d^{2} \varphi_{-}\right)}^{2} \\
& \quad+\left\|2 \partial_{-} g\left(\varphi_{+}^{2}, \varphi_{-}^{2}\right)\right\|_{L^{p}\left(\mathbb{R}^{2} \times \mathbb{R}^{2}, d^{2} \varphi_{+} d^{2} \varphi_{-}\right)}^{2}+\left\|4 \partial_{+} \partial_{-} g\left(\varphi_{+}^{2}, \varphi_{-}^{2}\right)\right\|_{L^{p}\left(\mathbb{R}^{2} \times \mathbb{R}^{2}, d^{2} \varphi_{+} d^{2} \varphi_{-}\right)}^{2},
\end{aligned}
$$

with $\partial_{ \pm} g\left(\varphi_{+}^{2}, \varphi_{-}^{2}\right)=\partial / \partial \varphi_{ \pm}^{2} g\left(\varphi_{+}^{2}, \varphi_{-}^{2}\right)$. We have $[20,21] \xi_{\lambda, z} \in \mathscr{K}_{\infty}$ for all $\lambda \in \mathbb{R}$ and $z=E+i \eta$ with $\eta>0$. The map $(\lambda, E, \eta) \rightarrow \xi_{\lambda, E+i \eta}$ is continuous from $\mathbb{R} \times$ $\mathbb{R} \times(0, \infty)$ to $\mathscr{K}_{\infty}$. If $|E|<\sqrt{K}$ we have $\xi_{0, E} \in \mathscr{K}_{\infty}$ and $\lim _{\eta \downarrow 0} \xi_{0, E+i \eta}=\xi_{0, E}$ in $\mathscr{K}_{\infty}$. (Notice that although $\xi_{0, E}$ can be defined for any $E \in \mathbb{R}$ by a pointwise limit, as in (3.4), $\xi_{0, E}$ is in the Banach space $\mathscr{K}_{\infty}$ if and only if $|E|<\sqrt{K}$.)

We will also use the Hilbert space $\mathscr{L}$, defined as the completion of

$$
\begin{aligned}
& \{g:[0, \infty) \times[0, \infty) \rightarrow \mathbb{C} \text { measurable; } \\
& \left.\quad\|g\|_{\mathscr{L}} \equiv\left\|g\left(\varphi_{+}^{2}, \varphi_{-}^{2}\right)\right\|_{L^{2}\left(\mathbb{R}^{2} \times \mathbb{R}^{2}, d^{2} \varphi_{+} d^{2} \varphi_{-}\right)}<\infty\right\} .
\end{aligned}
$$

We can identify $\mathscr{L}$ with the subspace of $L^{2}\left(\mathbb{R}^{2} \times \mathbb{R}^{2}, d^{2} \varphi_{+} d^{2} \varphi_{-}\right)$consisting of functions depending only on $\varphi_{+}^{2}$ and $\varphi_{-}^{2}$, and define $\mathscr{F}$ as the restriction to $\mathscr{L}$ of the Fourier transform on $L^{2}\left(\mathbb{R}^{2} \times \mathbb{R}^{2}, d^{2} \varphi_{+} d^{2} \varphi_{-}\right)$, so $\mathscr{F}$ is the unitary operator on 
$\mathscr{L}$ given by

$$
(\mathscr{F} g)\left(\varphi_{+}^{2}, \varphi_{-}^{2}\right)=\frac{1}{4 \pi^{2}} \int_{\mathbb{R}^{2} \times \mathbb{R}^{2}} \mathrm{e}^{-i\left(\varphi_{+} \cdot \varphi_{+}^{\prime}-i \varphi_{-} \cdot \varphi_{-}^{\prime}\right)} g\left(\varphi_{+}^{\prime}{ }^{2}, \varphi_{-}^{\prime}{ }^{2}\right) d^{2} \varphi_{+}^{\prime} d^{2} \varphi_{-}^{\prime} .
$$

We define linear operators

$$
\mathscr{T}=4 \mathscr{F} \partial_{+} \partial_{-}
$$

and

$$
\mathscr{B}_{\lambda, z}=M\left(\mathrm{e}^{i E\left(\varphi_{+}^{2}-\varphi_{-}^{2}\right)-\eta\left(\varphi_{+}^{2}+\varphi_{-}^{2}\right)} h\left(\lambda\left(\varphi_{+}^{2}-\varphi_{-}^{2}\right)\right)\right),
$$

where $M\left(g\left(\varphi_{+}^{2}, \varphi_{-}^{2}\right)\right)$ denotes multiplication by the function $g\left(\varphi_{+}^{2}, \varphi_{-}^{2}\right)$. It turns out that $\mathscr{T}$ is unitary on $\mathscr{K}_{2}$ [7] and a bounded linear operator from $\mathscr{K}_{1}$ to $\mathscr{K}_{\infty}, \mathscr{B}_{\lambda, z}$ is a bounded linear operator on all $\mathscr{K}_{p}$, and $g \rightarrow g^{n}$ is a continuous map from $\mathscr{K}_{\infty}$ to $\mathscr{K}_{1}$ for any $n=2,3, \ldots$. Moreover, $\xi_{\lambda, z}$ satisfies the nonlinear equation in $\mathscr{K}_{\infty}$ :

$$
\xi_{\lambda, z}=\mathscr{T} \mathscr{B}_{\lambda, z} \xi_{\lambda, z}^{K}
$$

not only for all $\lambda \in \mathbb{R}$ and $z=E+i \eta$ with $\eta>0$, but also for $\eta=0$ if $\lambda=0$ and $|E|<\sqrt{K}$. (Equation (3.10) can be verified by writing explicitly the right-hand side and performing all integrations.)

The following result is proven in $[20,21]$ and will play a major role in the proof of Theorem 2.2.

Theorem 3.1. For any $E$ such that $|E|<\sqrt{K}$ there exist $\lambda_{E}>0$ and $\varepsilon_{E}>0$, such that the map $\left(\lambda, E^{\prime}, \eta\right) \rightarrow \xi_{\lambda, E^{\prime}+i \eta} \in \mathscr{K}_{\infty}$, defined for

$$
\begin{gathered}
\left(\lambda, E^{\prime}, \eta\right) \in\left(\left[\left(-\lambda_{E}, \lambda_{E}\right) \times\left(E-\varepsilon_{E}, E+\varepsilon_{E}\right) \times(0, \infty)\right]\right. \\
\left.\cup\left[\{0\} \times\left(E-\varepsilon_{E}, E+\varepsilon_{E}\right) \times\{0\}\right]\right),
\end{gathered}
$$

has a continuous extension to $\left(-\lambda_{E}, \lambda_{E}\right) \times\left(E-\varepsilon_{E}, E+\varepsilon_{E}\right) \times[0, \infty)$ satisfying (3.10).

\section{Proof of Theorem 2.2}

We start from the equation

$$
\mathbb{E}\left(\left|G_{\lambda}(0, x ; z)\right|^{2}\right)=\left\langle\xi_{\lambda, z} \mid \mathscr{V}_{\lambda, z}^{|x|} \xi_{\lambda, z}\right\rangle_{\lambda, z}
$$

where

$$
\begin{aligned}
\langle f \mid g\rangle_{\lambda, z} & =\frac{1}{\pi^{2}} \int_{\mathbb{R}^{2} \times \mathbb{R}^{2}} f\left(\varphi_{+}^{2}, \varphi_{-}^{2}\right)\left[\mathscr{B}_{\lambda, z} M\left(\xi_{\lambda, z}^{K-1}\right) g\right]\left(\varphi_{+}^{2}, \varphi_{-}^{2}\right) d^{2} \varphi_{+} d^{2} \varphi_{-} \\
& =\frac{1}{\pi^{2}}\left\langle\bar{f}, \mathscr{B}_{\lambda, z} M\left(\xi_{\lambda, z}^{K-1}\right) g\right\rangle_{\mathscr{L}}
\end{aligned}
$$

is a bounded symmetric bilinear form on $\mathscr{L}$, and hence on $\mathscr{K}_{\infty}$, and $\mathscr{V}_{\lambda, z}=$ $\mathscr{F} \mathscr{B}_{\lambda, z} M\left(\xi_{\lambda, z}^{K-1}\right)$ is easily seen to be a contraction operator on $\mathscr{L}$. A derivation of (4.1) is given in Appendix B. 
Thus

$$
\begin{aligned}
J_{\lambda}(E+i \eta) & \equiv \sum_{x \in \mathbb{B}}|x|^{2} \mathbb{E}\left(\left|G_{\lambda}(0, x ; E+i \eta)\right|^{2}\right) \\
& =\frac{K+1}{K} \sum_{r=1}^{\infty} r^{2}\left\langle\xi_{\lambda, z} \mid \mathscr{W}_{\lambda, z}^{r} \xi_{\lambda, z}\right\rangle
\end{aligned}
$$

where $\mathscr{W}_{\lambda, z}=K \mathscr{V}_{\lambda, z}$.

Lemma 4.1. Let $\lambda \in \mathbb{R}, z=E+i \eta$ with $E \in \mathbb{R}$ and $\eta>0$.

(i) For all $f, g \in \mathscr{L}$,

$$
\left\langle f \mid \mathscr{W}_{\lambda, z} g\right\rangle_{\lambda, z}=\left\langle\mathscr{W}_{\lambda, z} f \mid g\right\rangle_{\lambda, z}
$$

(ii) $\mathscr{W}_{\lambda, z}^{2}$ is a compact operator.

(iii) Let

$$
\theta_{\lambda, z}=-2\left(\partial_{+}+\partial_{-}\right) \xi_{\lambda, z} .
$$

Then $\theta_{\lambda, z} \in \mathscr{L}$ and

$$
\mathscr{W}_{\lambda, z} \theta_{\lambda, z}=\theta_{\lambda, z}-\frac{4 \eta}{K} \mathscr{W}_{\lambda, z} \xi_{\lambda, z}
$$

(iv) For any $r=0,1,2, \ldots$ we have

$$
\left\langle\xi_{\lambda, z} \mid \mathscr{W}_{\lambda, z}^{r} \theta_{\lambda, z}\right\rangle_{\lambda, z}>\left\langle\xi_{\lambda, z} \mid \mathscr{W}_{\lambda, z}^{r+1} \theta_{\lambda, z}\right\rangle_{\lambda, z}>0
$$

and

$$
\left\langle\theta_{\lambda, z} \mid \mathscr{W}_{\lambda, z}^{r} \theta_{\lambda, z}\right\rangle_{\lambda, z}>\left\langle\theta_{\lambda, z} \mid \mathscr{W}_{\lambda, z}^{r+1} \theta_{\lambda, z}\right\rangle_{\lambda, z}>0
$$

Notice that (4.8) is in [31].

Proof. (i) follows from $\mathscr{F}$ being unitary on $\mathscr{L}$ where we have $\overline{\mathscr{F} f}=\mathscr{F} \bar{f}$. (ii) is a consequence of $\mathscr{B}_{\lambda, z} \mathscr{F} \mathscr{B}_{\lambda, z}$ being a compact operator in $\mathscr{L}$ for $\eta>0$, which can be shown as in [25, Proposition 9(i)].

To prove (iii), we use (3.10) and (3.8) to get

$$
\theta_{\lambda, z}=-2\left(\partial_{+}+\partial_{-}\right) \mathscr{T} \mathscr{B}_{\lambda, z} \xi_{\lambda, z}^{K}=-2\left(\partial_{+}+\partial_{-}\right)\left[4 \mathscr{F} \partial_{+} \partial_{-}\left\{\mathscr{B}_{\lambda, z} \xi_{\lambda, z}^{K}\right\}\right] .
$$

Since $[7,25]$

$$
4 \partial_{ \pm} \mathscr{F} \partial_{ \pm}=\mathscr{F}
$$

we have

$$
\theta_{\lambda, z}=\mathscr{F}\left[-2\left(\partial_{+}+\partial_{-}\right)\left\{\mathscr{B}_{\lambda, z} \xi_{\lambda, z}^{K}\right\}\right]=\mathscr{W}_{\lambda, z} \theta_{\lambda, z}+\frac{4 \eta}{K} \mathscr{W}_{\lambda, z} \xi_{\lambda, z}
$$

as

$$
\begin{gathered}
-2\left(\partial_{+}+\partial_{-}\right)\left(\mathrm{e}^{i E\left(\varphi_{+}^{2}-\varphi_{-}^{2}\right)-\eta\left(\varphi_{+}^{2}+\varphi_{-}^{2}\right)} h\left(\lambda\left(\varphi_{+}^{2}-\varphi_{-}^{2}\right)\right)\right) \\
=4 \eta\left(\mathrm{e}^{i E\left(\varphi_{+}^{2}-\varphi_{-}^{2}\right)-\eta\left(\varphi_{+}^{2}+\varphi_{-}^{2}\right)} h\left(\lambda\left(\varphi_{+}^{2}-\varphi_{-}^{2}\right)\right)\right) .
\end{gathered}
$$

We prove that

$$
\left\langle\xi_{\lambda, z} \mid \mathscr{W}_{\lambda, z}^{r} \theta_{\lambda, z}\right\rangle_{\lambda, z}>0 \text { and }\left\langle\theta_{\lambda, z} \mid \mathscr{W}_{\lambda, z}^{r} \theta_{\lambda, z}\right\rangle_{\lambda, z}>0
$$


in Appendix B. Using (4.8), we have

$$
\begin{aligned}
\left\langle\xi_{\lambda, z} \mid \mathscr{W}_{\lambda, z}^{r+1} \theta_{\lambda, z}\right\rangle_{\lambda, z} & =\left\langle\xi_{\lambda, z} \mid \mathscr{W}_{\lambda, z}^{r} \theta_{\lambda, z}\right\rangle_{\lambda, z}-\frac{4 \eta}{K}\left\langle\xi_{\lambda, z} \mid \mathscr{W}_{\lambda, z}^{r+1} \xi_{\lambda, z}\right\rangle_{\lambda, z} \\
& <\left\langle\xi_{\lambda, z} \mid \mathscr{W}_{\lambda, z}^{r} \theta_{\lambda, z}\right\rangle_{\lambda, z}
\end{aligned}
$$

since $\left\langle\xi_{\lambda, z} \mid \mathscr{W}_{\lambda, z}^{r+1} \xi_{\lambda, z}\right\rangle_{\lambda, z}>0$ by (4.1). Similarly,

$$
\begin{aligned}
\left\langle\theta_{\lambda, z} \mid \mathscr{W}_{\lambda, z}^{r+1} \theta_{\lambda, z}\right\rangle_{\lambda, z} & =\left\langle\theta_{\lambda, z} \mid \mathscr{W}_{\lambda, z}^{r} \theta_{\lambda, z}\right\rangle_{\lambda, z}-\frac{4 \eta}{K}\left\langle\theta_{\lambda, z} \mid \mathscr{W}_{\lambda, z}^{r+1} \xi_{\lambda, z}\right\rangle_{\lambda, z} \\
& <\left\langle\theta_{\lambda, z} \mid \mathscr{W}_{\lambda, z}^{r} \theta_{\lambda, z}\right\rangle_{\lambda, z}
\end{aligned}
$$

since $\left\langle\theta_{\lambda, z} \mid \mathscr{W}_{\lambda, z}^{r+1} \xi_{\lambda, z}\right\rangle_{\lambda, z}>0$ by (4.15) and (4.16). Thus (iv) is proven.

Lemma 4.2. Let $\lambda \in \mathbb{R}, z=E+$ in with $E \in \mathbb{R}$ and $\eta>0$. Then

$$
\begin{aligned}
J_{\lambda}(E+i \eta)= & \frac{(K+1)}{4 \eta}\left\langle\xi_{\lambda, z} \mid \theta_{\lambda, z}\right\rangle_{\lambda, z}+\frac{K(K+1)}{16 \eta^{2}} \\
& \times\left(\left\langle\theta_{\lambda, z} \mid \theta_{\lambda, z}\right\rangle_{\lambda, z}+2 \sum_{r=0}^{\infty}\left\langle\theta_{\lambda, z} \mid \mathscr{W}_{\lambda, z}^{r} \theta_{\lambda, z}\right\rangle_{\lambda, z}\right)
\end{aligned}
$$

Proof. Using (4.8), we have

$$
\begin{aligned}
& \sum_{r=1}^{R} r^{2}\left\langle\xi_{\lambda, z} \mid \mathscr{W}_{\lambda, z}^{r} \xi_{\lambda, z}\right\rangle_{\lambda, z}=\frac{K}{4 \eta} \sum_{r=1}^{R} r^{2}\left\langle\xi_{\lambda, z} \mid \mathscr{W}_{\lambda, z}^{r-1}\left(I-\mathscr{W}_{\lambda, z}\right) \theta_{\lambda, z}\right\rangle_{\lambda, z} \\
& \quad=\frac{K}{4 \eta}\left\{\sum_{r=0}^{R-1}(2 r+1)\left\langle\xi_{\lambda, z} \mid \mathscr{W}_{\lambda, z}^{r} \theta_{\lambda, z}\right\rangle_{\lambda, z}-R^{2}\left\langle\xi_{\lambda, z} \mid \mathscr{W}_{\lambda, z}^{R} \theta_{\lambda, z}\right\rangle_{\lambda, z}\right\}
\end{aligned}
$$

Since $\mathscr{W}_{\lambda, z}^{2}$ is a compact operator and $\left\langle\xi_{\lambda, z} \mid \mathscr{W}_{\lambda, z}^{r} \theta_{\lambda, z}\right\rangle_{\lambda, z}$ is not constant and has a limit as $r \rightarrow \infty$ by (4.9), we can apply Theorem C.1 to conclude that $\left\langle\xi_{\lambda, z}\right| \mathscr{W}_{\lambda, z}^{r}$ $\left.\theta_{\lambda, z}\right\rangle_{\lambda, z} \rightarrow 0$ exponentially fast, so we have

$$
J_{\lambda}(E+i \eta)=\frac{K+1}{4 \eta} \sum_{r=0}^{\infty}(2 r+1)\left\langle\xi_{\lambda, z} \mid \mathscr{W}_{\lambda, z}^{r} \theta_{\lambda, z}\right\rangle_{\lambda, z}
$$

Repeating the procedure, we get

$$
\begin{aligned}
& \sum_{r=1}^{R}(2 r+1)\left\langle\xi_{\lambda, z} \mid \mathscr{W}_{\lambda, z}^{r} \theta_{\lambda, z}\right\rangle_{\lambda, z}=\frac{K}{4 \eta} \sum_{r=1}^{R}(2 r+1)\left\langle\left(I-\mathscr{W}_{\lambda, z}\right) \theta_{\lambda, z} \mid \mathscr{W}_{\lambda, z}^{r-1} \theta_{\lambda, z}\right\rangle_{\lambda, z} \\
& \quad=\frac{K}{4 \eta}\left\{\left\langle\theta_{\lambda, z} \mid \theta_{\lambda, z}\right\rangle_{\lambda, z}+2 \sum_{r=0}^{R-1}\left\langle\theta_{\lambda, z} \mid \mathscr{W}_{\lambda, z}^{r} \theta_{\lambda, z}\right\rangle_{\lambda, z}-(2 R+1)\left\langle\theta_{\lambda, z} \mid \mathscr{W}_{\lambda, z}^{R} \theta_{\lambda, z}\right\rangle_{\lambda, z}\right\}
\end{aligned}
$$

As before, we use (4.10), the compactness of $\mathscr{W}_{\lambda, z}^{2}$ and Theorem C.1 to let $R \rightarrow \infty$ in (4.25). Combining the resulting expression with (4.25) we get (4.20).

We now need the following extensions of (4.8). 
Lemma 4.3. Let $\lambda \in \mathbb{R}, z=E+$ i with $E \in \mathbb{R}$ and $\eta>0$. Then for any $r=$ $1,2, \ldots$ we have

$$
\mathscr{W}_{\lambda, z}^{r} \theta_{\lambda, z}=\theta_{\lambda, z}-\frac{4 \eta}{K} \sum_{s=1}^{r} \mathscr{W}_{\lambda, z}^{s} \xi_{\lambda, z}
$$

and

$$
\begin{aligned}
\left\langle\theta_{\lambda, z} \mid \mathscr{W}_{\lambda, z}^{r} \theta_{\lambda, z}\right\rangle_{\lambda, z}= & \left\langle\theta_{\lambda, z} \mid \theta_{\lambda, z}\right\rangle_{\lambda, z}-\frac{4 \eta}{K} r\left\langle\xi_{\lambda, z} \mid \theta_{\lambda, z}\right\rangle_{\lambda, z} \\
& +\frac{16 \eta^{2}}{K^{2}} \sum_{s=1}^{r}(r-s+1)\left\langle\xi_{\lambda, z} \mid \mathscr{W}_{\lambda, z}^{s} \xi_{\lambda, z}\right\rangle_{\lambda, z}
\end{aligned}
$$

Proof. Equation (4.26) follows immediately from (4.28) by induction. Using (4.26) twice, we get

$$
\begin{aligned}
& \left\langle\theta_{\lambda, z} \mid \mathscr{W}_{\lambda, z}^{r} \theta_{\lambda, z}\right\rangle_{\lambda, z}=\left\langle\theta_{\lambda, z} \mid \theta_{\lambda, z}\right\rangle_{\lambda, z}-\frac{4 \eta}{K}\left\langle\xi_{\lambda, z} \mid \sum_{u=1}^{r} \mathscr{W}_{\lambda, z}^{u} \theta_{\lambda, z}\right\rangle_{\lambda, z} \\
& =\left\langle\theta_{\lambda, z} \mid \theta_{\lambda, z}\right\rangle_{\lambda, z}-\frac{4 \eta}{K} r\left\langle\xi_{\lambda, z} \mid \theta_{\lambda, z}\right\rangle_{\lambda, z}+\frac{16 \eta^{2}}{K^{2}} \sum_{u=1}^{r} \sum_{s=1}^{u}\left\langle\xi_{\lambda, z} \mid \mathscr{W}_{\lambda, z}^{s} \xi_{\lambda, z}\right\rangle_{\lambda, z},
\end{aligned}
$$

so (4.27) follows.

Lemma 4.4. Let $\lambda \in \mathbb{R}, z=E+$ i $\eta$ with $E \in \mathbb{R}$ and $\eta>0$. Then

$$
\begin{aligned}
J_{\lambda}(E+i \eta) \geqq & \frac{(K+1)}{4 \eta}\left\langle\xi_{\lambda, z} \mid \theta_{\lambda, z}\right\rangle_{\lambda, z}+\frac{3 K(K+1)}{16 \eta^{2}}\left\langle\theta_{\lambda, z} \mid \theta_{\lambda, z}\right\rangle_{\lambda, z} \\
& +\frac{K^{2}(K+1)}{64 \eta^{3}} \frac{\left(\left\langle\theta_{\lambda, z} \mid \theta_{\lambda, z}\right\rangle_{\lambda, z}-\frac{4 \eta}{K}\left\langle\xi_{\lambda, z} \mid \theta_{\lambda, z}\right\rangle_{\lambda, z}\right)^{2}}{\left\langle\xi_{\lambda, z} \mid \theta_{\lambda, z}\right\rangle_{\lambda, z}}
\end{aligned}
$$

Proof. From (4.27) and (4.15) we have

$$
\begin{aligned}
\sum_{r=1}^{\infty}\left\langle\theta_{\lambda, z} \mid \mathscr{W}_{\lambda, z}^{r} \theta_{\lambda, z}\right\rangle_{\lambda, z} & \geqq \sum_{r=1}^{\infty}\left(\left\{\left\langle\theta_{\lambda, z} \mid \theta_{\lambda, z}\right\rangle_{\lambda, z}-\frac{4 \eta}{K} r\left\langle\xi_{\lambda, z} \mid \theta_{\lambda, z}\right\rangle_{\lambda, z}\right\} \vee 0\right) \\
& =\sum_{r=1}^{R_{\lambda, z}}\left(\left\langle\theta_{\lambda, z} \mid \theta_{\lambda, z}\right\rangle_{\lambda, z}-\frac{4 \eta}{K} r\left\langle\xi_{\lambda, z} \mid \theta_{\lambda, z}\right\rangle_{\lambda, z}\right) \\
& \geqq \frac{K\left(\left\langle\theta_{\lambda, z} \mid \theta_{\lambda, z}\right\rangle_{\lambda, z}-\frac{4 \eta}{K}\left\langle\xi_{\lambda, z} \mid \theta_{\lambda, z}\right\rangle_{\lambda, z}\right)^{2}}{8 \eta\left\langle\xi_{\lambda, z} \mid \theta_{\lambda, z}\right\rangle_{\lambda, z}}
\end{aligned}
$$


where

$$
R_{\lambda, z}=\left[\frac{K\left\langle\theta_{\lambda, z} \mid \theta_{\lambda, z}\right\rangle_{\lambda, z}}{4 \eta\left\langle\xi_{\lambda, z} \mid \theta_{\lambda, z}\right\rangle_{\lambda, z}}\right]
$$

with $x \vee y$ the maximum of $x$ and $y$ and $[x]$ the largest integer $\leqq x$.

Equation (4.29) now follows from (4.20) and (4.32).

We are ready to prove Theorem 2.2. If $|E|<\sqrt{K}$, (3.4) gives

$$
\theta_{0, E}=\frac{2 \sqrt{K-E^{2}}}{K} \xi_{0, E}
$$

and

$$
\left\langle\xi_{0, E} \mid \xi_{0, E}\right\rangle_{0, E}=\left|G_{0}(0,0 ; E)\right|^{2}=\frac{4 K}{(K+1)^{2}-4 E^{2}}>\frac{4 K}{(K-1)^{2}}
$$

Thus

$$
\left\langle\xi_{0, E} \mid \theta_{0, E}\right\rangle_{0, E}=\frac{8 \sqrt{K-E^{2}}}{(K+1)^{2}-4 E^{2}}>0
$$

and

$$
\left\langle\theta_{0, E} \mid \theta_{0, E}\right\rangle_{0, E}=\frac{16\left(K-E^{2}\right)}{K\left((K+1)^{2}-4 E^{2}\right)}>0 .
$$

We now use Theorem 3.1, for each $E$ such that $|E|<\sqrt{K}$ we can pick $\lambda_{E}>0$ and $\varepsilon_{E}>0$ so the map $\left(\lambda, E^{\prime}, \eta\right) \rightarrow \xi_{\lambda, E^{\prime}+i \eta} \in \mathscr{K}_{\infty}$ has a continuous extension to $\left(-\lambda_{E}, \lambda_{E}\right) \times\left(E-\varepsilon_{E}, E+\varepsilon_{E}\right) \times[0, \infty)$ satisfying (3.10). It follows that the map $\left(\lambda, E^{\prime}, \eta\right) \rightarrow \theta_{\lambda, E^{\prime}+\text { in }} \in \mathscr{L}$ also has a continuous extension to $\left(-\lambda_{E}, \lambda_{E}\right) \times$ $\left(E-\varepsilon_{E}, E+\varepsilon_{E}\right) \times[0, \infty)$ and (4.7) holds. In addition, the complex valued maps $\left(\lambda, E^{\prime}, \eta\right) \rightarrow\left\langle\xi_{\lambda, E^{\prime}+i \eta} \mid \theta_{\lambda, E^{\prime}+i \eta}\right\rangle_{\lambda, E^{\prime}+i \eta}$ and $\left(\lambda, E^{\prime}, \eta\right) \rightarrow\left\langle\theta_{\lambda, E^{\prime}+i \eta} \mid \theta_{\lambda, E^{\prime}+i \eta}\right\rangle_{\lambda, E^{\prime}+i \eta}$ have continuous extensions to $\left(-\lambda_{E}, \lambda_{E}\right) \times\left(E-\varepsilon_{E}, E+\varepsilon_{E}\right) \times[0, \infty)$ such that

$$
\left\langle\xi_{\lambda, E^{\prime}+i \eta} \mid \theta_{\lambda, E^{\prime}+i \eta}\right\rangle_{\lambda, E^{\prime}+i \eta}>0 \quad \text { and } \quad\left\langle\theta_{\lambda, E^{\prime}+i \eta} \mid \theta_{\lambda, E^{\prime}+i \eta}\right\rangle_{\lambda, E^{\prime}+i \eta}>0
$$

if $\lambda_{E}>0$ and $\varepsilon_{E}>0$ were chosen sufficiently small.

Theorem 2.2 now follows from (4.29).

\section{A. Time Evolution and Green's Functions}

In this appendix we prove some useful facts. We consider the Hamiltonian

$$
H_{\lambda}=\frac{1}{2} \Delta+V \quad \text { on } l^{2}(\mathbb{L})
$$

where $V$ is a fixed potential; we define $r^{2}(t)$ as in (1.4).

We start with a result of Simon [35].

Lemma A.1. For any $t \in \mathbb{R}$ we have

$$
r^{2}(t) \leqq\left\|\frac{1}{2} \Delta\right\|^{2} t^{2}
$$


Proof. Let $|x|$ denote also the operator multiplication by $|x|$. We have

$$
r^{2}(t)=\left\||x| \mathrm{e}^{i t H} \delta_{0}\right\|^{2}=\left\|\mathrm{e}^{-i t H}|x| \mathrm{e}^{i t H} \delta_{0}\right\|^{2} .
$$

Using the Fundamental Theorem of Calculus we get

$$
r^{2}(t)=\left\|\int_{0}^{t} \mathrm{e}^{-i s H}[H,|x|] \mathrm{e}^{i s H} \delta_{0} d s\right\|^{2} \leqq\left\|\left[\frac{1}{2} \Delta,|x|\right]\right\|^{2} t^{2} \leqq\left\|\frac{1}{2} \Delta\right\|^{2} t^{2} .
$$

The next well known lemma gives the Laplace transform of $r^{2}(t)$ in terms of the Green's function $G(x, y ; z)=\left\langle\delta_{x},(H-z)^{-1} \delta_{y}\right\rangle$ (e.g., $[14,28]$ ).

Lemma A.2. For any $\eta>0$ we have

$$
\int_{0}^{\infty} \mathrm{e}^{-\eta t} r^{2}(t) d t=\frac{1}{2 \pi} \int_{-\infty}^{\infty}\left\{\sum_{x \in \mathbb{L}}|x|^{2}\left|G\left(0, x ; E+i \frac{\eta}{2}\right)\right|^{2}\right\} d E .
$$

Proof. By the spectral theorem,

$$
\left(H-E+i \frac{\eta}{2}\right)^{-1}=\int_{0}^{\infty} \mathrm{e}^{i t E} \mathrm{e}^{-i t\left(H-i \frac{\eta}{2}\right)} d t .
$$

Taking matrix elements of both sides and applying Plancherel's Theorem we get

$$
\int_{0}^{\infty} \mathrm{e}^{-\eta t}\left|\mathrm{e}^{i t H}(0, x)\right|^{2} d t=\frac{1}{2 \pi} \int_{-\infty}^{\infty}\left|G\left(0, x ; E+i \frac{\eta}{2}\right)\right|^{2} d E .
$$

The lemma follows.

From (A.7) we obtain

$$
\int_{-\infty}^{\infty}\left|G\left(0, x ; E+i \frac{\eta}{2}\right)\right|^{2} d E \leqq \frac{2 \pi}{\eta} .
$$

Combining Lemmas A.1 and A.2 we get

$$
\int_{-\infty}^{\infty}\left\{\sum_{x \in \mathbb{L}}|x|^{2}\left|G\left(0, x ; E+i \frac{\eta}{2}\right)\right|^{2}\right\} d E \leqq \frac{4 \pi}{\eta^{3}}\left\|\frac{1}{2} \Delta\right\|^{2} .
$$

This last estimate should be compared with

$$
\sup _{E \in \mathbb{R}} \sum_{x \in \mathbb{L}}|x|^{2}\left|G\left(0, x ; E+i \frac{\eta}{2}\right)\right|^{2} \leqq \frac{4}{\eta^{4}}\left\|\frac{1}{2} \Delta\right\|^{2},
$$

which follows from Lemma A.1 and the inequality

$$
\begin{aligned}
\sum_{x \in \mathbb{L}}|x|^{2}\left|G\left(0, x ; E+i \frac{\eta}{2}\right)\right|^{2} & =\left\||x|\left(H-\left(E+i \frac{\eta}{2}\right)\right)^{-1} \delta_{0}\right\|^{2} \\
& =\left\|\int_{0}^{\infty}|x| \mathrm{e}^{i t E} \mathrm{e}^{-i t\left(H-i \frac{\eta}{2}\right)} d t\right\|^{2} \\
& \leqq\left(\int_{0}^{\infty} \mathrm{e}^{-\frac{\eta}{2} t}\left[r^{2}(t)\right]^{\frac{1}{2}} d t\right)^{2}
\end{aligned}
$$




\section{B. The Supersymmetric Replica Trick}

In this appendix we derive (4.1) and (4.15), using the supersymmetric replica trick (see $[18,24,31])$.

Let $\Lambda$ be a finite subset of $\mathbb{B}$, we will use $H_{\lambda, \Lambda}$ to denote the operator $H_{\lambda}$ restricted to $l^{2}(\Lambda)$ with Dirichlet boundary conditions. The corresponding Green's function is

$$
\begin{aligned}
G_{\lambda, \Lambda}(x, y ; z)= & \left\langle\delta_{x},\left(H_{\lambda, \Lambda}-z\right)^{-1} \delta_{y}\right\rangle, \quad x, y \in \Lambda \\
& \text { and } z=E+i \eta \text { with } E \in \mathbb{R}, \eta>0 .
\end{aligned}
$$

The supersymmetric replica trick (see [18]) says that if $x_{1}, x_{2} \in \Lambda$, then for all $z \in \mathbb{C}$ with $\eta=\operatorname{Im} z>0$, we have

$$
G_{\lambda, \Lambda}\left(x_{1}, x_{2} ; z\right)=i \int \psi\left(x_{1}\right) \bar{\psi}\left(x_{2}\right) \exp \left\{-i \sum_{x \in \Lambda} \Phi(x) \cdot\left[\left(H_{\lambda, \Lambda}-z\right) \Phi\right](x)\right\} D_{\Lambda} \Phi,
$$

where $\Phi(x)=(\varphi(x), \psi(x), \bar{\psi}(x))$ with $\varphi(x) \in \mathbb{R}^{2}$ and $\psi(x), \bar{\psi}(x)$ anticommuting "variables" (i.e., elements of a Grassmann algebra),

$$
\Phi(x) \cdot \Phi(y)=\varphi(x) \cdot \varphi(y)+\frac{1}{2}(\bar{\psi}(x) \psi(y)+\bar{\psi}(y) \psi(x))
$$

and

$$
D_{\Lambda} \Phi=\prod_{x \in \Lambda} d \Phi(x) \quad \text { with } \quad d \Phi(x)=\frac{1}{\pi} d \bar{\psi}(x) d \psi(x) d^{2} \varphi(x) .
$$

To compute functions of $\psi, \bar{\psi}$ we expand in a power series that terminates after a finite number of terms due to the anticommutativity. The linear functional denoted by integration against $d \bar{\psi}(x) d \psi(x)$ is defined by

$$
\int\left(a_{0}+a_{1} \psi(x)+a_{2} \bar{\psi}(x)+a_{3} \bar{\psi}(x) \psi(x)\right) d \bar{\psi}(x) d \psi(x)=-a_{3} .
$$

We will use the (bad, but convenient) notation $\Phi(x)^{2}=\Phi(x) \cdot \Phi(x)$ and $\varphi(x)^{2}=$ $\varphi(x) \cdot \varphi(x)$. We will also denote a generic $\Phi(x)=(\varphi(x), \psi(x), \bar{\psi}(x))$ by $\Phi=$ $(\varphi, \psi, \bar{\psi})$. Notice that if $f:[0, \infty) \rightarrow \mathbb{C}$ is continuously differentiable, then $f\left(\Phi^{2}\right)$ $=f\left(\varphi^{2}\right)+f^{\prime}\left(\varphi^{2}\right) \bar{\psi} \psi$.

The lattice $\mathbb{B}_{l}$ will consist of all sites in $\mathbb{B}$ whose distance from the fixed origin 0 is less than or equal to $l$; similarly $\mathbb{B}_{l}^{(x \mid y)}, \mathbb{B}_{l}^{(x)}$ will denote all sites in $\mathbb{B}^{(x \mid y)}, \mathbb{B}^{(x)}$, respectively, whose distance from $x$ is less than or equal to $l$. (For convenience we also allow $l=\infty$, in which case it may be omitted from the notation.) We will

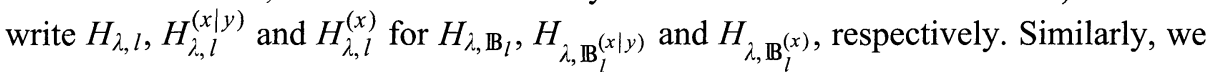
will use $G_{\lambda, l}(x, y ; z)$ for $G_{\lambda, \mathbb{B}_{l}}(x, y ; z)$ and $G_{\lambda, l}(z), G_{\lambda, l}^{(x \mid y)}(z), G_{\lambda, l}^{(x)}(z)$ for $G_{\lambda, l}(0,0 ; z)$,

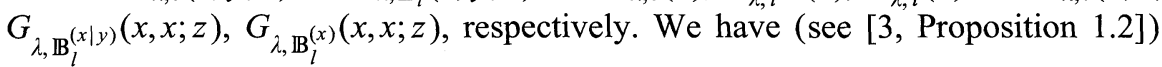

$$
\lim _{l \rightarrow \infty} G_{\lambda, l}(x, y ; z)=G_{\lambda}(x, y ; z) \text { for any } x, y \in \mathbb{B}, E \in \mathbb{R} \text { and } \eta>0,
$$

with similar limits for $G_{\lambda, l}(z), G_{\lambda, l}^{(x \mid y)}(z)$ and $G_{\lambda, l}^{(x)}(z)$.

We now fix $x \in \mathbb{B}$ and set $r=|x| \equiv d(x, 0)$. Let $x_{0}, x_{1}, \ldots, x_{r}$ be the shortest path from 0 to $x$, i.e., $x_{0}=0, x_{r}=x$ and $d\left(x_{n}, x_{n+1}\right)=1$ for $n=0,1, \ldots, r-1$, 
with $x_{n} \neq x_{n^{\prime}}$ for $n \neq n^{\prime}$. For each $n=0,1, \ldots, r-1$, let $J_{n}=\{1, \ldots, K-1\}$ for $n=$ $1, \ldots, r-1$ and $J_{n}=\{0,1, \ldots, K-1\}$ for $n=0, r$; we write $\left\{x_{n}^{(j)} ; j \in J_{n}\right\}$ for the collection of nearest neighbors sites to $x_{n}$ which are not in the path $x_{0}, x_{1}, \ldots, x_{r}$. By $\mathbb{B}_{x, l}$ we denote the lattice consisting of all sites in $\mathbb{B}$ whose distance from the path $x_{0}, x_{1}, \ldots, x_{r}$ is at most $l$; notice that as a set

$$
\mathbb{B}_{x, l}=\left\{x_{0}, x_{1}, \ldots, x_{r}\right\} \cup\left[\bigcup_{n=0}^{r} \bigcup_{j \in J_{n}} \mathbb{B}_{l}^{\left(x_{n}^{(j)} \mid x_{n}\right)}\right]
$$

We write $H_{\lambda, x, l}$ and $G_{\lambda, x, l}(0, x ; z)$ for $H_{\lambda, \mathbb{B}_{x, l}}$ and $G_{\lambda, \mathbb{B}_{x, l}}(0, x ; z)$, respectively. As in (B.4), we have

$$
\lim _{l \rightarrow \infty} G_{\lambda, x, l}(0, x ; z)=G_{\lambda}(0, x ; z) .
$$

If $l<\infty$ are finite and $\operatorname{Im} z>0$, (B.2) gives

$$
G_{\hat{\lambda}, x, l}(0, x ; z)=i \int \psi(0) \bar{\psi}(x)\left\{\prod_{n=0}^{r-1} \mathrm{e}^{-i \Phi\left(x_{n}\right) \cdot \Phi\left(x_{n+1}\right)}\right\}\left\{\prod_{n=0}^{r} \Xi_{n}\right\} D_{\mathbb{B}_{x, l}} \Phi,
$$

where

$$
\begin{aligned}
& \Xi_{n}=\mathrm{e}^{i\left(z-\lambda V\left(x_{n}\right)\right) \Phi\left(x_{n}\right)^{2}} \\
& \quad \exp \left\{-i \sum_{j \in J_{n}}\left(\Phi\left(x_{n}\right) \cdot \Phi\left(x_{n}^{(j)}\right)+\sum_{y \in \mathbb{B}_{l}^{\left(x_{n}^{(j)} \mid x_{n}\right)}} \Phi(y) \cdot\left[\left(H_{\lambda, l}^{\left(x_{n}^{(j)} \mid x_{n}\right)}-z\right) \Phi\right](y)\right)\right\}
\end{aligned}
$$

By an explicit computation,

$$
\begin{aligned}
& \int \exp \left\{-i\left(\Phi\left(x_{n}\right) \cdot \Phi\left(x_{n}^{(j)}\right)+\sum_{y \in \mathbb{B}_{l}^{\left(x_{n}^{(j)} \mid x_{n}\right)}} \Phi(y) \cdot\left[\left(H_{\lambda, l}^{\left(x_{n}^{(j)} \mid x_{n}\right)}-z\right) \Phi\right](y)\right)\right\} D_{\mathbb{B}_{l}^{\left(x_{n}^{(j)} \mid x_{n}\right)}} \Phi \\
& \quad=\exp \left\{\frac{i}{4} G_{\lambda, l}^{\left(x_{n}^{(j)} \mid x_{n}\right)}(z) \Phi\left(x_{n}\right)^{2}\right\} .
\end{aligned}
$$

Thus

$$
\begin{aligned}
G_{\lambda, x, l}(0, x ; z)= & i \int \psi(0) \bar{\psi}(x)\left\{\prod_{n=0}^{r-1} \mathrm{e}^{-i \Phi\left(x_{n}\right) \cdot \Phi\left(x_{n+1}\right)}\right\} \\
& \times\left\{\prod_{n=0}^{r} \Upsilon_{l, n}\left(\Phi\left(x_{n}\right)^{2}\right)\right\} \prod_{n=0}^{r} d \Phi\left(x_{n}\right),
\end{aligned}
$$

where

$$
\Upsilon_{l, n}\left(\varphi^{2}\right)=\exp \left\{i\left[z-\lambda V\left(x_{n}\right)+\frac{1}{4} \sum_{j \in J_{n}} G_{\lambda, l}^{\left(x_{n}^{())} \mid x_{n}\right)}(z)\right] \varphi^{2}\right\}, \quad n=0,1, \ldots, r .
$$


We now let $l \rightarrow \infty$, obtaining

$$
\begin{aligned}
G_{\lambda}(0, x ; z)= & i \int \psi(0) \bar{\psi}(x)\left\{\prod_{n=0}^{r-1} \mathrm{e}^{-i \Phi\left(x_{n}\right) \cdot \Phi\left(x_{n+1}\right)}\right\} \\
& \times\left\{\prod_{n=0}^{r} \Upsilon_{n}\left(\Phi\left(x_{n}\right)^{2}\right)\right\} \prod_{n=0}^{r} d \Phi\left(x_{n}\right) .
\end{aligned}
$$

We can now integrate over the anticommuting variables, getting

$$
\begin{aligned}
G_{\lambda}(0, x ; z)= & \frac{i}{\pi} \int\left\{\prod_{n=0}^{r-1} \frac{i}{2 \pi} \mathrm{e}^{-i \varphi\left(x_{n}\right) \cdot \varphi\left(x_{n+1}\right)}\right\} \\
& \times\left\{\prod_{n=0}^{r} \Upsilon_{n}\left(\varphi\left(x_{n}\right)^{2}\right)\right\} \prod_{n=0}^{r} d^{2} \varphi\left(x_{n}\right) .
\end{aligned}
$$

Thus

$$
\begin{aligned}
\left|G_{\lambda}(0, x ; z)\right|^{2}= & \frac{1}{\pi^{2}} \int \prod_{n=0}^{r-1} \frac{1}{(2 \pi)^{2}} \mathrm{e}^{-i\left(\varphi_{+}\left(x_{n}\right) \cdot \varphi_{+}\left(x_{n+1}\right)-\varphi_{-}\left(x_{n}\right) \cdot \varphi_{-}\left(x_{n+1}\right)\right)} \\
& \times \prod_{n=0}^{r} \Gamma_{n}\left(\varphi_{+}\left(x_{n}\right)^{2}, \varphi_{-}\left(x_{n}\right)^{2}\right) \prod_{n=0}^{r} d^{2} \varphi_{+}\left(x_{n}\right) d^{2} \varphi_{-}\left(x_{n}\right),
\end{aligned}
$$

where

$$
\Gamma_{n}\left(\varphi_{+}^{2}, \varphi_{-}^{2}\right)=\Upsilon_{n}\left(\varphi_{+}^{2}\right) \overline{\Upsilon_{n}\left(\varphi_{-}^{2}\right)} .
$$

Since the $\{V(x) ; x \in \mathbb{B}\}$ are independent random variables, we have

$$
\begin{aligned}
\mathbb{E}\left(\left|G_{\lambda}(0, x ; z)\right|^{2}\right)= & \frac{1}{\pi^{2}} \int \prod_{n=0}^{r-1} \frac{1}{(2 \pi)^{2}} \mathrm{e}^{-i\left(\varphi_{+}\left(x_{n}\right) \cdot \varphi_{+}\left(x_{n+1}\right)-\varphi_{-}\left(x_{n}\right) \cdot \varphi_{-}\left(x_{n+1}\right)\right)} \\
& \times \prod_{n=0}^{r} \mathbb{E}\left(\Gamma_{n}\left(\varphi_{+}^{2}, \varphi_{-}^{2}\right)\right) \prod_{n=0}^{r} d^{2} \varphi_{+}\left(x_{n}\right) d^{2} \varphi_{-}\left(x_{n}\right),
\end{aligned}
$$

with

$$
\mathbb{E}\left(\Gamma_{n}\left(\varphi_{+}^{2}, \varphi_{-}^{2}\right)\right)=\mathrm{e}^{i E\left(\varphi_{+}^{2}-\varphi_{-}^{2}\right)-\eta\left(\varphi_{+}^{2}+\varphi_{-}^{2}\right)} h\left(\lambda\left(\varphi_{+}^{2}-\varphi_{-}^{2}\right)\right)\left(\xi_{\lambda_{, z}}\left(\varphi_{+}^{2}, \varphi_{-}^{2}\right)\right)^{\left|J_{n}\right|},
$$

where $\left|J_{n}\right|=K-1$ if $n=1, \ldots, r-1$ and $\left|J_{n}\right|=K$ for $n=0, r$, where $\xi_{\lambda, z}$ is defined in (3.1).

A moment of reflexion now reveals that (4.1) is simply an elegant (and useful) way of rewriting (B.15). Moreover, we have from (3.1) and (4.7) that

$$
\theta_{\lambda, z}=\mathbb{E}\left(\mathscr{I}_{\lambda}^{(0)}(z) \exp \left\{\frac{i}{4}\left(G_{\lambda}^{(0)}(z) \varphi_{+}^{2}-\overline{G_{\lambda}^{(0)}(z)} \varphi_{-}^{2}\right)\right\}\right) .
$$

Proceeding as in the derivation of (B.15), we get

$$
\left\langle\theta_{\lambda, z} \mid \mathscr{V}_{\lambda, z}^{|x|} \xi_{\lambda, z}\right\rangle_{\lambda, z}=\mathbb{E}\left(\mathscr{I}_{\lambda}^{\left(0^{(0)} \mid 0\right)}(z)\left|G_{\lambda}(0, x ; z)\right|^{2}\right)>0
$$

and

$$
\left\langle\theta_{\lambda, z} \mid \mathscr{V}_{\lambda, z}^{r} \theta_{\lambda, z}\right\rangle_{\lambda, z}=\mathbb{E}\left(\mathscr{I}_{\lambda}^{\left(0^{(0)} \mid 0\right)}(z) \mathscr{I}_{\lambda}^{\left(x^{(0)} \mid x\right)}(z)\left|G_{\lambda}(0, x ; z)\right|^{2}\right)>0,
$$

proving (4.15). 


\section{Some Spectral Analysis}

Let $\mathscr{X}$ be a Banach space. A bounded linear operator $A$ on $\mathscr{X}$ will be said to be of type $K$ if $A$ has discrete spectrum outside some closed set contained in the open unit disk in the complex plane, i.e., we have

$$
\sigma(A)=\Lambda \cup\left\{\lambda_{1}, \lambda_{2}, \ldots, \lambda_{k}\right\},
$$

where $\Lambda \subset D=\{z \in \mathbb{C} ;|z|<1\}$ is closed in $\mathbb{C}$ and $\left\{\lambda_{1}, \lambda_{2}, \ldots, \lambda_{k}\right\} \subset \mathbb{C} \backslash D$ is a finite collection of isolated eigenvalues with finite multiplicity. Powers of compact operators and, more generally, Riesz operators are of type $K$.

Theorem C.1. Let $A$ be a bounded linear operator of type $K$ on the Banach space $\mathscr{X}$ and let us fix $x \in \mathscr{X}$ and $l \in \mathscr{X}^{*}$. We set

$$
\alpha(n)=l\left(A^{n} x\right) \text { for } n=1,2, \ldots .
$$

If $\alpha(n)$ is not constant in $n$ and $\lim _{n \rightarrow \infty} \alpha(n)$ exists in $\mathbb{C}$, then $\alpha(n) \rightarrow 0$ exponentially fast as $n \rightarrow \infty$, i.e., there exist $C<\infty$ and $\gamma>0$ such that

$$
|\alpha(n)| \leqq C \mathrm{e}^{-\gamma n} \quad \text { for all } n=1,2, \ldots .
$$

Proof. By redefining $\Lambda$ we can assume $\left|\lambda_{i}\right| \geqq 1$ in (C.1) for all $i=1,2, \ldots, k$. It follows from (C.1) that (see [17, Sect. III.6])

$$
A=R+\sum_{i=1}^{k}\left(\lambda_{i} P_{i}+D_{i}\right)
$$

where $R$ and $P_{i}, D_{i}, i=1,2, \ldots, k$, are bounded linear operators on $\mathscr{X}$ such that:

$$
P_{i} P_{j}=\delta_{i j} P_{i} \quad \text { for all } i, j=1,2, \ldots, k .
$$

(ii) For each $i=1,2, \ldots, k$,

$$
P_{i} D_{i}=D_{i} P_{i}=D_{i}
$$

and

$$
D_{i}^{h_{i}}=0 \text { for some positive integer } h_{i}
$$

(iii)

$$
\sigma(R)=\Lambda
$$

and

$$
P_{i} R=R P_{i}=0 \quad \text { for each } i=1,2, \ldots, k
$$

It follows that

$$
A^{n}=R^{n}+\sum_{i=1}^{k}\left(\lambda_{i} P_{i}+D_{i}\right)^{n} \quad \text { for all } n=1,2, \ldots
$$

Since the spectral radius of $R$ is $<1, l\left(R^{n} x\right)$ can be bounded as in (C.3). Thus it suffices to consider the case when $R=0$, in which case we will show that $\alpha(n)$ does not have a limit in $\mathbb{C}$ as $n \rightarrow \infty$, unless it is constant in $n$. 
From (C.6) and (C.7) we have

$$
\left(\lambda_{i} P_{i}+D_{i}\right)^{n}=\lambda_{i}^{n} P_{i}+\sum_{s=1}^{h_{i}-1}\left(\begin{array}{l}
n \\
s
\end{array}\right) \lambda_{l}^{n-s} D_{i}^{s},
$$

for any $n \geqq h_{i}-1$. It then follows that

$$
l\left(\left(\lambda_{i} P_{i}+D_{i}\right)^{n} x\right)=\sum_{s=0}^{h_{l}-1}\left(\begin{array}{l}
n \\
s
\end{array}\right) \lambda_{i}^{n-s} a_{i, s}
$$

for some complex numbers $a_{i, s}, s=0,1, \ldots, h_{i}-1$. If they are not all zero, we can choose $s_{i} \in\left\{0,1, \ldots, h_{i}-1\right\}$ such that

$$
l\left(\left(\lambda_{i} P_{i}+D_{i}\right)^{n} x\right)=\left(\begin{array}{c}
n \\
s_{i}
\end{array}\right) \lambda_{i}^{n}\left(b_{i}+\beta_{i}(n)\right)
$$

for some complex numbers $b_{i} \neq 0$ and $\beta_{i}(n)$ with $\lim _{n \rightarrow \infty} \beta_{i}(n)=0$.

Thus, if $R=0$ and $\alpha(n) \neq 0$, we have, for all $n \geqq \sup _{i=1,2, \ldots, k}\left(h_{i}-1\right)$, that

$$
\alpha(n)=\left(\begin{array}{l}
n \\
s
\end{array}\right) r^{n}\left(\sum_{j=1}^{t} c_{j} \mathrm{e}^{i 2 \pi \theta_{j} n}+\beta(n)\right),
$$

for some $r \geqq 1$, nonnegative integer $s$, positive integer $t$, distinct $\theta_{J} \in[0,1), j=$ $1,2, \ldots, t$, and $\beta(n)$ with $\lim _{n \rightarrow \infty} \beta(n)=0$. If $t=1$, we have $\lim _{n \rightarrow \infty} \alpha(n)=\infty$, unless $r=1$ and $s=0$, in which case we also have $\beta(n)=0$, so $\alpha(n)$ is either constant or has no limit as $n \rightarrow \infty$. If $t>1$, we use Lemma C.2 below to conclude that $\limsup _{n \rightarrow \infty}|\alpha(n)|=\infty$, unless $r=1$ and $s=0$, in which case $|\alpha(n)|$ is bounded but $\alpha(n)$ has no limit as $n \rightarrow \infty$.

Lemma C.2. Let $\theta_{j} \in[0,1), j=1,2, \ldots, t$ be distinct $(t>1)$ and $c_{j}, j=1,2, \ldots, t$ be nonzero complex numbers. Then $\Gamma(n) \equiv \sum_{j=1}^{t} c_{j} \mathrm{e}^{i 2 \pi \theta_{j} n}$ has no limit as $n \rightarrow \infty$.

Proof. If $\theta_{j} \in[0,1), j=1,2, \ldots, t$ are all rationals, then the range of $\Gamma(n)$ consists of a finite number (at least 2, since $t>1$ ) of values, each taken infinitely many times. It thus suffices to prove that if $\theta_{j} \in[0,1), j=1,2, \ldots, t$ are all irrationals, then $\Gamma(n)$ has an infinite number of distinct limit points as $n \rightarrow \infty$.

So suppose $\theta_{j} \in[0,1), j=1,2, \ldots, t$ are all irrationals. Relabeling the indices if necessary, we can find $u \in\{1,2, \ldots, t\}$ such that $1, \theta_{1}, \theta_{2}, \ldots, \theta_{u}$ are linearly independent over the integers, and there exist linear functions $f_{j}$ on $\mathbb{R}^{(u+1)}$ with integer coefficients, $j=u+1, u+2, \ldots, t$, such that $\theta_{j}=f_{j}\left(1, \theta_{1}, \theta_{2}, \ldots, \theta_{u}\right)$ for $j=$ $u+1, u+2, \ldots, t$. We define the complex valued function $F$ on the $u$-dimensional torus $\mathbb{T}^{u}$ by

$$
\begin{aligned}
F\left(\varphi_{1}, \varphi_{2}, \ldots, \varphi_{u}\right)= & \sum_{j=1}^{t} c_{j} \mathrm{e}^{i 2 \pi \varphi_{j}} \quad \text { with } \varphi_{j}=f_{j}\left(1, \varphi_{1}, \varphi_{2}, \ldots, \varphi_{u}\right) \\
& \text { for } j=u+1, u+2, \ldots, t
\end{aligned}
$$

We also let $\tau$ be the rotation on $\mathbb{T}^{u}$ given by

$$
\tau\left(\varphi_{1}, \varphi_{2}, \ldots, \varphi_{u}\right)=\left(\varphi_{1}+\theta_{1}, \varphi_{2}+\theta_{2}, \ldots, \varphi_{u}+\theta_{u}\right),
$$


all sums being $\bmod 1$. As the $f_{j}, j=u+1, u+2, \ldots, t$, are linear functions, we have

$$
\Gamma(n)=F\left(\tau^{n}(0,0, \ldots, 0)\right) .
$$

Since $1, \theta_{1}, \theta_{2}, \ldots, \theta_{u}$ are linearly independent over the integers, $\tau$ is a minimal ergodic transformation on $\mathbb{T}^{u}$, and hence the orbit of any point in $\mathbb{T}^{u}$ visits each neighborhood infinitely many times. $F$ being continuous, it follows that any point on its range is a limit point for $\Gamma(n)$ as $n \rightarrow \infty$. But the range of $F$ is a connected compact subset of $\mathbb{C}$, which is clearly not a single point, and hence has infinite cardinality.

\section{Some Elementary Probability}

Lemma D.1. Let $X_{n}$ be a sequence of random variables such that:

1. With probability one

$$
0 \leqq X_{n} \leqq M<\infty \text { for all } n \text {. }
$$

2.

$$
\limsup _{n \rightarrow \infty} \mathbb{E}\left(X_{n}\right)>0
$$

Then

$$
\mathbb{P}\left(\limsup _{n \rightarrow \infty} X_{n}>0\right)>0 .
$$

Proof. By passing to a subsequence we assume $\mathbb{E}\left(X_{n}\right)>a$ for all $n$ and some $a>0$. We have

$$
\begin{aligned}
a & <\mathbb{E}\left(X_{n} ; X_{n}>\frac{a}{2}\right)+\mathbb{E}\left(X_{n} ; X_{n} \leqq \frac{a}{2}\right) \\
& \leqq M \mathbb{P}\left(X_{n}>\frac{a}{2}\right)+\frac{a}{2} \mathbb{P}\left(X_{n} \leqq \frac{a}{2}\right) \\
& =\left(M-\frac{a}{2}\right) \mathbb{P}\left(X_{n}>\frac{a}{2}\right)+\frac{a}{2},
\end{aligned}
$$

so

$$
\mathbb{P}\left(X_{n}>\frac{a}{2}\right)>\frac{a}{2 M-a}>0 .
$$

We now define the events

$$
A_{n}=\left\{X_{n}>\frac{a}{2}\right\}
$$

and

$$
A=\limsup _{n \rightarrow \infty} A_{n} .
$$

It now follows from (D.7) that

$$
\mathbb{P}(A) \geqq \limsup _{n \rightarrow \infty} \mathbb{P}\left(A_{n}\right) \geqq \frac{a}{2 M-a}>0 .
$$

Since

the lemma is proven.

$$
A \subset\left\{\limsup _{n \rightarrow \infty} X_{n}>0\right\},
$$


Acknowledgements. It is a pleasure to thank Svetlana Jitomirskaya for many useful discussions and Lemma D.1.

\section{References}

1. Abou-Chacra, R., Anderson, P., Thouless, D.J.: A selfconsistent theory of localization. J. Phys. C: Solid State Phys. 6, 1734-1752 (1973)

2. Abou-Chacra, P., Thouless, D.J.: Selfconsistent theory of localization: II. Localization near the band edges. J. Phys. C: Solid State Phys. 7, 65-75 (1974)

3. Acosta, V., Klein, A.: Analyticity of the density of states in the Anderson model in the Bethe lattice. J. Stat. Phys. 69, 277-305 (1992)

4. Aizenman, M.: Localization at weak disorder: Some elementary bounds. Rev. Math. Phys., to appear

5. Aizenman, M., Molchanov, S.: Localization at large disorder and extreme energies: An elementary derivation. Commun. Math. Phys. 157, 245-278 (1993)

6. Anderson, P.: Absence of diffusion in certain random lattices. Phys. Rev. 109, 1492-1505 (1958)

7. Campanino, M., Klein, A.: A supersymmetric transfer matrix and differentiability of the density of states in the one-dimensional Anderson model. Commun. Math. Phys. 104, 227-241 (1986)

8. Carmona, R., Klein, A., Martinelli, F.: Anderson localization for Bernoulli and other singular potentials. Commun. Math. Phys. 108, 41-66 (1987)

9. Carmona, R., Lacroix, J.: Spectral Theory of Random Schrödinger Operators. Boston, MA: Birkhäuser, 1990

10. Delyon, F., Levy, Y., Souillard, B.: Anderson localization for multidimensional systems at large disorder or low energy. Commun. Math. Phys. 100, 463-470 (1985)

11. von Dreifus, H., Klein, A.: A new proof of localization in the Anderson tight binding model. Commun. Math. Phys. 124, 285-299 (1989)

12. Figotin, A., Klein, A.: Localization phenomenon in gaps of the spectrum of random lattice operators. J. Stat. Phys. 75, 997-1021 (1994)

13. Fröhlich, J., Martinelli, F., Scoppola, E., Spencer, T.: Constructive proof of localization in the Anderson tight binding model. Commun. Math. Phys. 101, 21-46 (1985)

14. Fröhlich, J., Spencer, T.: Absence of diffusion in the Anderson tight binding model for large disorder or low energy. Commun. Math. Phys. 88, 151-184 (1983)

15. Gol'dsheid, Ya., Molchanov, S., Pastur, L.: Pure point spectrum of stochastic one dimensional Schrödinger operators. Funct. Anal. Appl. 11, 1-10 (1977)

16. Graf, G.M.: Anderson localization and the space-time characteristic of continuum states. J. Stat. Phys. 75, 337-346 (1994)

17. Kato, T.: Perturbation theory for linear operators. Berlin, Heidelberg, New York: Springer, 1966

18. Klein, A.: The supersymmetric replica trick and smoothness of the density of states for random Schrödinger operators. Proc. Symposia in Pure Mathematics 51, 315-331 (1990)

19. Klein, A.: Localization in the Anderson model with long range hopping. Braz. J. Phys. 23, 363-371 (1993)

20. Klein, A.: Absolutely continuous spectrum in the Anderson model on the Bethe lattice. Mathematical Research Letters 1, 399-407 (1994)

21. Klein, A.: Extended states in the Anderson model on the Bethe lattice. Advances in Math., to appear

22. Klein, A.: The Anderson metal-insulator transition on the Bethe lattice. In: Iagolnitzer, D. (ed.), Mathematical Physics XI, to appear

23. Klein, A.: Absolutely continuous spectrum in random Schrödinger operators. Proc. Symposia in Pure Mathematics, to appear

24. Klein, A., Martinelli, F., Perez, J.F.: A rigorous replica trick approach to Anderson localization in one dimension. Commun. Math. Phys. 106, 623-633 (1986)

25. Klein, A., Speis, A.: Regularity of the invariant measure and of the density of states in the one-dimensional Anderson model. J. Funct. Anal. 88, 211-227 (1990) 
26. Kunz, H., Souillard, B.: Sur le spectre des operateurs aux differences finies aleatoires. Commun. Math. Phys. 78, 201-246 (1980)

27. Kunz, H., Souillard, B.: The localization transition on the Bethe lattice. J. Phys. (Paris) Lett. 44, 411-414 (1983)

28. Martinelli, F., Scoppola, E.: Introduction to the mathematical theory of Anderson localization. Rivista del Nuovo Cimento 10 n. 10 (1987)

29. McKane, A. J., Stone, M.: Localization as an alternative to Goldstone's theorem. Ann. Phys. 131, 36-55 (1981)

30. Miller, J., Derrida, B.: Weak disorder expansion for the Anderson model on a tree. J. Stat. Phys. 75, 357-389 (1994)

31. Mirlin, A. D., Fyodorov, Y.V.: Localization transition in the Anderson model on the Bethe lattice: Spontaneous symmetry breaking and correlation functions. Nucl. Phys. B 366, 507-532 (1991)

32. Mott, N., Twose, W.: The theory of impurity conduction. Adv. Phys. 10, 107-163 (1961)

33. Pastur, L.: Spectra of random selfadjoint operators. Russ. Math. Surv. 28, 1-67 (1973)

34. Simon, B.: Functional integration and quantum physics. New York: Academic Press, 1979

35. Simon, B.: Absence of ballistic motion. Commun. Math. Phys. 134, 209-212 (1990)

36. Simon, B., Wolff, T.: Singular continuum spectrum under rank one perturbations and localization for random Hamiltonians. Commun. Pure. Appl. Math. 39, 75-90 (1986)

37. Thouless, D.: Electrons in disordered systems and the theory of localization. Phys. Rev. 13, 93-106 (1974)

Communicated by B. Simon 
